\title{
SOME CHARACTERIZATIONS OF REFLEXIVITY
}

\section{IVAN SINGER 1}

ABSTRACT. The results of R. C. James on characterizations of reflexivity of Banach spaces with an unconditional basis in terms of $c_{0}$ and $l^{1}$ are extended to arbitrary $B$ anach spaces. Some consequences are obtained.

R. C. James has proved [4, Theorem 2] that a Banach space $E$ with an unconditional basis is reflexive if and only if $E$ contains no subspace isomorphic to $c_{0}$ or $l^{1}$. This result has been shown to remain valid for any subspace $E$ of a space with an unconditional basis (of any power) by C. Bessaga and A. Pełczyński [1], [2], who have also proved [2] that such a space $E$ is reflexive if and only if $E^{*}$ contains no subspace isomorphic to $l^{1}$.

The above conditions are clearly necessary for the reflexivity of any Banach space $E$. In the present note we shall show that one can add a certain necessary condition to them, which is also satisfied by any (not necessarily reflexive) subspace of a space with an unconditional basis (of any power) in such a way that these conditions together will be also sufficient for reflexivity. Thus, we shall obtain extensions of the above results to characterizations of reflexivity of an arbitrary Banach space $E$.

Following A. PeXczyński [6], a Banach space $E$ is said to have property (u), if for every weak Cauchy sequence $\left\{x_{n}\right\} \subset E$ there exists a sequence $\left\{y_{n}\right\} \subset E$ such that (a) the series $\Sigma_{i=1}^{\infty} y_{i}$ is weakly unconditionally Cauchy and (b) the sequence $\left\{x_{n}-\sum_{i=1}^{n} y_{i}\right\}$ converges weakly to 0 。

Theorem.For a Banach space $E$ the following statements are equivalent:

$1^{\circ}$. E is reflexive. or $l^{1}$.

$2^{\circ}$. E has property $(\mathrm{u})$ and $E$ contains no subspace isomorphic to $c_{0}$

$3^{\circ}$. E has property (u) and $E^{*}$ contains no subspace isomorphic to $l^{1}$.

Proof Assume $1^{\circ}$. Then, by [6, Proposition 2], $E$ has property (u). Also, $E^{*}$ is reflexive and hence $E^{*}$ contains no subspace isomorphic to $l^{1}$. Thus, $1^{\circ} \Rightarrow 3^{\circ}$.

Received by the editors August 1, 1974.

AMS (MOS) subject classifications (1970). Primary 46B10.

${ }^{1}$ Prepared during the author's visit at Université de Montréal, on leave from the Institute of Mathematics of the Romanian Academy of Sciences. 
Assume now $3^{\circ}$. If $E$ contains a subspace $G$ isomorphic to $c_{0}$, then $E^{*}$ has a quotient space $E^{*} / G^{\perp}$ isomorphic to $l^{1}$ and hence (see e.g. [3, p. 63, exercise 2]) $E^{*}$ has a complemented subspace isomorphic to $l^{1}$, in contradiction with $3^{\circ}$. On the other hand, if $E$ contains a subspace isomorphic to $l^{1}$, then so does $E^{*}\left[7\right.$, Proposition 3.3], in contradiction with $3^{\circ}$. Thus, $3^{\circ} \Rightarrow 2^{\circ}$.

Assume now $2^{\circ}$. Then, since $E$ has property (u) and contains no subspace isomorphic to $c_{0}$, by [6, Theorem 1] (for a proof see [9, p. 450]), $E$ is weakly complete. Hence, since $E$ contains no subspace isomorphic to $l^{1}$, from [8, Corollary 1] it follows that $E$ is reflexive. Thus, $2^{\circ} \Rightarrow 1^{\circ}$.

Since every Banach space $E$ with an unconditional basis and every subspace of such a space have property (u) (by [6, Theorem 3 and Corollary 1]; for a proof see [9, pp. 445-449]), from the above Theorem we obtain, in particular, the results of R. C. James [4] and C. Bessaga and A. PeXczyński $[1],[2]$ mentioned in the introduction.

Corollary 1. A separable Banach space $E$ is reflexive if and only if (i) E has property (u) and (ii) $E^{* *}$ is separable.

Proof. Clearly, (i) and (ii) imply $3^{\circ}$ of the above Theorem.

Remark 1. Combining [8] with [6, Corollary 5], it follows that if a Banach space $E$ has property (u) and contains no subspace isomorphic to $l^{1}$, then $E^{*}$ is weakly complete. This result yields other proofs of Corollary 1 and the implication $3^{\circ} \Rightarrow 1^{\circ}$ of the Theorem.

Corollary 2. The following two conjectures are equivalent:

$1^{\circ}[5, \mathrm{p} .165]$. Every infinite dimensional Banach space contains an infinite dimensional subspace that is either reflexive or is isomorphic to $c_{0}$ or $l^{1}$.

$2^{\circ}$. Every infinite dimensional Banach space contains an infinite dimen. sional subspace with property $(\mathrm{u})$,

Proof. $c_{0}, l^{1}$ and every reflexive space have property (u), so $1^{\circ} \Rightarrow 2^{\circ}$. Conversely, if $G \subset E$ has property (u), then by the above Theorem either $G$ is reflexive or $G$ contains a subspace isomorphic to $c_{0}$ or $l^{1}$. Thus, $2^{\circ} \Rightarrow 1^{\circ}$.

Remark 2. The conjecture of Corollary 2, if substantiated, would have some interesting consequences, e.g., that every (infinite dimensional) sec. ond conjugate space $E^{* *}$ contains a reflexive subspace (of infinite dimension)-or, equivalently, that every conjugate Banach space $E^{*}$ has a reflexive quotient space. Indeed, if $E \supset G$ reflexive, then $E^{* *} \supset E \supset G$; if $E \supseteq$ $c_{0}$, then $E^{* *} \supseteq l^{\infty} \supset l^{2}$; finally, if $E \supseteq l^{1}$, then $E^{* *} \supseteq\left(l^{\infty}\right)^{*} \supset l^{2}$. 


\section{REFERENCES}

1. C. Bessaga and A. Pełczyński, A generalization of results of $R$. C. James concerning absolute bases in Banach spaces, Studia Math. 17 (1958), 165-174. MR $22 \# 5874$.

2. - On subspaces of a space with an absolute basis, Bull. Acad. Polon. Sci. Sér. Sci. Math. Astronom. Phys. 6 (1958), 313-315. MR 20 \# 3438.

3. A. Grothendieck, Espaces vectoriels topologiques, 2 nd ed., Instituto Matemática Pura e Aplicada, Universidade de São Paulo, São Paulo, 1958.

4. R. C. James, Bases and reflexivity of Banach spaces, Ann. of Math. (2) 52 (1950), 518-527. MR 12, 616.

5. J. Lindenstrauss, Some aspects of the theory of Banach spaces, Advances in Math. 5 (1970), 159-180. MR 43 \# 5288.

6. A. Pełczyński, A connection between weakly unconditional convergence and weakly completeness of Banach spaces, Bull. Acad. Polon. Sci. Sér. Sci. Math. Astronom. Phys. 6 (1958), 251-253. MR 22 \#5875.

7. - On Banach spaces containing $L_{1}(\mu)$, Studia Math. 30 (1968), 231246. MR $38 \# 521$.

8. H. P. Rosenthal, A characterization of Banach spaces containing $l^{1}$, Proc. Nat. Acad. Sci. U. S. A. 71 (1974), 2411-2413.

9. I. Singer, Bases in Banach spaces. I, Die Grundlehren der math. Wissenschaften, Band 154, Springer-Verlag, New York and Berlin, 1970. MR 45 \#4451.

DÉPARTEMENT D'INFORMATIQUE, UNIVERSITÉ DE MONTRÉAL, MONTRÉAL, CANADA

INSTITUTE OF MATHEMATICS, ACADEMY OF SCIENCES, BUCHAREST, ROMANIA 